\title{
Inequalities relating to some Algebraic Means.
}

By R. F. Moirhead, M.A., B.Sc.

The fact that for two or more real positive quantities there exist three well-known algebraic means, the Arithmetic, the Geometric, and the Harmonic, which stand in a fixed order of magnitude independent of the quantities operated on, suggests the question whether there may not be other algebraic means that stand in a definite order of magnitude with reference to those just named and to one another. The following paper supplies an affirmative answer to the question. The results given in the first section are, so far as I know, novel; some of those in the second section are well known, but I hope some freshness may be apparent in their treatment here.

I.

1. Let there be $n$ positive quantities $a, b, c \ldots$, and let ${ }_{n} \mathbf{P}_{p}$ denote the sum of the products of all possible $p$-combinations of $a, b, c \ldots$, and ${ }_{n} \mathrm{C}_{p}$ the number of these, and let ${ }_{n} \mathrm{~A}_{p}$ denote the quotient ${ }_{n} \mathrm{P}_{p} / \mathrm{C}_{p}$; then the quantities

$$
{ }_{n} \mathbf{A}_{1}, \quad\left({ }_{n} \mathbf{A}_{2}\right)^{\frac{1}{2}}, \quad\left({ }_{n} \mathbf{A}_{3}\right)^{\frac{1}{3}}, \quad \ldots \ldots\left({ }_{n} \mathbf{A}_{p}\right)^{\frac{1}{\mu}} \ldots \ldots\left({ }_{n} \mathbf{A}_{n}\right)^{\frac{1}{n}}
$$

are all means of the $n$ given quantities, ${ }_{n} A_{1}$ being the arithmetic mean and $\left({ }_{n} A_{n}\right)^{\frac{1}{n}}$ the geometric mean. To fix ideas, we may note that ${ }_{n} \mathrm{P}_{1},{ }_{n} \mathrm{P}_{2} \ldots{ }_{n} \mathrm{P}_{n-1},{ }_{n} \mathrm{P}_{n}$ are the coefficients of $x^{n-1} x^{n-2} \ldots x, 1$ in the expansion of the product $(x+a)(x+b)(x+c) \ldots \ldots$ and that this expansion may also be written

$$
x^{n}+n_{n} \mathbf{A}_{1} x^{n-1}+\frac{n(n-1)}{1.2}{ }_{n} \mathrm{~A}_{2} x^{n-2}+\ldots+n_{n} \mathbf{A}_{n-1} x+{ }_{n} \mathbf{A}_{n} \text {. }
$$

We shall. show that the $n$ means above indicated are in descending order of magnitude when $a, b, c \ldots$ are not all equal; while of course each nean becomes $=a$ when $a=b=c \ldots$.

2. We shall first investigate the difference ${ }_{n} A_{p}^{p+1}-{ }_{n} A_{p+1}^{p}$ which we shall denote by ${ }_{n} \Delta_{p}$, using the simplified forms $A_{p}, P_{p}, C_{p}, \Delta_{p}$, etc., to denote ${ }_{n-1} \mathrm{~A}_{p},{ }_{n-1} \mathrm{P}_{k},{ }_{n-1} \mathrm{C}_{p},{ }_{n-1} \Delta_{p}$, the letters without prefix referring to the $n-1$ quaintities $b, c, d \ldots$. 
We shall prove that if $A_{1}, A_{2}^{\frac{1}{2}}, A_{3}^{\frac{1}{3}}, \ldots A_{n-1}^{\frac{1}{n-1}}$ are in descending order, then ${ }_{n} A_{1},{ }_{n} A_{2}^{\frac{1}{2}},{ }_{n} A_{3}^{\frac{1}{3}} \cdots{ }_{n} A_{n}^{\frac{1}{n}}$ are so also.

Assume in fact that $A_{p \rightarrow 1}^{\frac{1}{p-1}}>A_{p}^{\frac{1}{p}}>A_{p+1}^{\frac{1}{p+1}}$, and use the simpler symbol $m$ to denote the mean $A_{p}^{\frac{1}{p}}$, and we have

$$
\begin{aligned}
& \mathbf{P}_{p-1}=A_{p-1} \cdot \mathbf{C}_{p-1}>m^{p-1} \cdot \mathbf{C}_{p-1} \\
& \mathbf{P}_{p+1}=\mathbf{A}_{p+1} \quad \mathbf{C}_{p+1}<m^{p+1} \mathrm{C}_{p+1} \\
& \mathbf{P}_{p}=m^{p} \quad \mathbf{C}_{p}
\end{aligned}
$$

Thus ${ }_{n} \Delta_{p} \cdot{ }_{n} \mathrm{C}_{p+1}^{p} \cdot{ }_{n} \mathrm{C}_{p}^{p+1}$

$$
\begin{aligned}
& ={ }_{n} \mathrm{C}_{p+1}^{p}{ }_{n} \mathrm{P}_{p}^{p+1}-{ }_{n} \mathrm{C}_{p}^{p+1}{ }_{n} \mathrm{P}_{p+1}^{p} \\
& ={ }_{n} \mathrm{C}_{p+1}^{p}\left(a \mathrm{P}_{p-1}+\mathrm{P}_{p}\right)^{p+1}-{ }_{n} \mathrm{C}_{p}^{p+2}\left(a \mathrm{P}_{p}+\mathrm{P}_{p+1}\right)^{p} \\
& >{ }_{n} \mathrm{C}_{p+1}^{p}\left(a m^{p-1} \mathrm{C}_{\mu-1}+m^{p} \mathrm{C}_{p}\right)^{\mu+1}-{ }_{n} \mathrm{C}_{p}^{p+1}\left(a m^{p} \mathrm{C}_{p}+m^{p+1} \mathrm{C}_{p+1}\right)^{p}
\end{aligned}
$$

Thus ${ }_{n} \Delta_{p}>\left(a m^{p-1} \frac{\mathrm{C}_{p-1}}{{ }_{n} \mathrm{C}_{p}}+m^{\mu} \frac{\mathrm{C}_{p}}{{ }_{n} \mathrm{C}_{p}}\right)^{\nu+1}-\left(a m^{\nu} \frac{\mathrm{C}_{p}}{{ }_{n} \mathrm{C}_{p+1}}+m^{p+1} \frac{\mathrm{C}_{p+1}}{{ }^{\prime} \mathrm{C}_{p+1}}\right)^{p}$

$$
\begin{aligned}
& >m^{p^{2}-1}\left(a \frac{p}{n}+m \frac{n-p}{n}\right)^{p+1}-m^{p^{2}}\left(a \frac{p+1}{n}+m \frac{n-p-1}{n}\right)^{p} \\
& >\frac{m^{p^{2}-1}}{n^{p+1}}\left[\{(a-m) p+m n\}^{p+1}-m n\{(a-m)(p+1)+m n\}^{p}\right]
\end{aligned}
$$

Thus $n^{p+1}{ }_{n} \Delta_{p}>m^{p^{2}-1}\left[(v+m-a)^{p+1}-\{v+(p+1)(m-a)\} v^{p}\right]$

$$
\begin{aligned}
& \text { where } v \equiv(a-m)(p+1)+m n \\
& >m^{\prime \prime \prime}-1 v^{n+1}\left[(1+\beta)^{\mu+1}-1-(p+1) \beta\right] \\
& \text { where } \beta \equiv(m-a) / v \\
& >m^{i j-1} v^{\nu+1} \delta \text {. }
\end{aligned}
$$

Thus $\delta$

$$
\begin{aligned}
= & (1+\beta)^{p+1}-1-(p+1) \beta \\
= & (1+\beta-1)\left\{(1+\beta)^{p}+(1+\beta)^{p-1}+\ldots+(1+\beta)+1\right\}-(p+1) \beta \\
= & \beta\left\{(1+\beta)^{p}-1+(1+\beta)^{p-1}-1+\ldots+(1+\beta)-1+1-1\right\} \\
= & \beta^{2}\left[\left\{(1+\beta)^{p-1}+(1+\beta)^{p-2}+. .+1\right\}+\left\{(1+\beta)^{p-2}+(1+\beta)^{p-3}+\ldots+1\right\}\right. \\
& \quad+. .+\{(1+\beta)+1\}+1] \\
= & \beta^{2}\left[(1+\beta)^{p-1}+2(1+\beta)^{p-2}+3(1+\beta)^{p-3}+. .+(p-1)(1+\beta)+p\right] \ldots(4)
\end{aligned}
$$


This is essentially positive, since

$$
1+\beta=1+\frac{m-a}{m n+(a-m) p+1}=\frac{m(n-p)+p a}{m(n-p-1)+(p+1) a}
$$

which is never negative, $p+1$ being equal to $n$ at most.

Hence ${ }_{n} \Delta_{n}$ is positive if $A_{p-1}^{\frac{1}{p-1}}>A_{, !}^{\frac{1}{1}}>A_{p+1}^{j+\frac{1}{j+1}}$.

3. Now for $n=2$, we have ${ }_{2} A_{1}{ }^{2}-{ }_{2} A_{2}=\left(\frac{a+b}{2}\right)^{2}-a b=\frac{(a-b)^{2}}{4}$.

Again, for $n=3$, we have

$$
\begin{aligned}
{ }_{3} \mathbf{A}_{1}{ }^{2}-{ }_{3} \mathbf{A}_{2} & =\left(\frac{a+b+c}{3}\right)^{2}-\frac{b c+c a+a b}{3} \\
& =\frac{1}{18}\left\{(a-b)^{2}+(b-c)^{2}+(c-a)^{2}\right\}
\end{aligned}
$$

and ${ }_{3} \mathrm{~A}_{2}{ }^{3}-{ }_{3} \mathrm{~A}_{3}{ }^{2}=\left(\frac{b c+c a+a b}{3}\right)^{3}-(a b c)^{2}$

$$
\begin{array}{r}
=\frac{1}{54}\left[(a-b)^{2}\left\{7 a b c^{2}+(a+b) c^{3}\right\}+(b-c)^{2}\left\{7 b c a^{2}+(b+c) a^{3}\right\}\right. \\
\left.+(c-a)^{2}\left\{7 c a^{2}+(c+a) b^{3}\right\}\right] .
\end{array}
$$

Thus ${ }_{3} A_{1}>{ }_{3} A_{2} \frac{1}{2}>{ }_{3} A_{3} \frac{1}{3}$.

Hence by the result of the previous paragraph, similar inequalities hold for $n=4$, therefore for $n=5$, and so on.

Thus the inequalities are established for all values of $n$. It is true that in proving the inequalities at the two extremities of each series by the above method, we make use of the quantities

$$
{ }_{n-1} \mathrm{P}_{0},{ }_{n-1} \mathrm{P}_{n}, \quad{ }_{n-1} \mathrm{C}_{0}, \quad{ }_{n-1} \mathrm{C}_{n} \text {; }
$$

which as yet are not defined, but by taking them to mean $1,0,1,0$, the proof that ${ }_{n} \Delta_{p}$ is positive holds for $p=n-1$ and $p=1$ also.

But special proofs for these cases are easily found, and may be written as follows :

4. We have ${ }_{n} \Delta_{n-1}=\left(\frac{{ }_{n} P_{n-1}}{n}\right)^{n}-(a b c \ldots)^{n-1}$

$$
=(a b c \ldots)^{n}\left\{\frac{1}{n^{n}}\left(\frac{1}{a}+\frac{1}{b}+\ldots\right)^{n}-\frac{1}{a b c \ldots}\right\}
$$

which is positive, since the arithmetic mean of $\frac{1}{a}, \frac{1}{b}, \ldots$ is greater than their geometric mean. 


$$
\begin{aligned}
& \text { Again }{ }_{n} \Delta_{1}=\left(\frac{a+b+c+\ldots}{n}\right)^{2}-\frac{a b+a c+\ldots}{\frac{n(n-1)}{1.2}} \\
& =\frac{1}{n^{2}(n-1)}\left\{(n-1)(a+b+\ldots)^{2}-2 n(a b+a c+\ldots)\right\} \\
& =\frac{1}{n^{2}(n-1)}\left\{(n-1) \Sigma a^{2}-2 \Sigma a b\right\} \\
& =\frac{1}{n^{2}(n-1)}\left\{\mathscr{Y}\left(a^{2}+\dot{b^{2}}\right)-2 \Sigma a b ; \quad \text { since } \mathscr{Y}\left(a^{2}+b^{2}\right)=(n-1) \Sigma a^{2}\right. \\
& =\frac{1}{n^{2}(n-1)} \Sigma(a-b)^{2} \text {, which is positive. }
\end{aligned}
$$

5. Thus it appears that lying between the Arithmetic and Geometric Means of $n$ positive quantities there exist $n-2$ other means

$$
{ }_{n} A_{2}^{\frac{1}{2}} \quad{ }_{n} A_{3}^{\frac{1}{3}} \ldots{ }_{n} A_{n-1}^{n-1}
$$

which form a regularly descending series from the one to the other.

If the quantity ${ }_{n} \mathrm{H}_{1}$ defined by the equation

$$
\frac{n}{{ }_{n} \mathrm{H}_{1}}=\frac{1}{a}+\frac{1}{b}+\frac{1}{c}+\ldots . .
$$

be called the Harmonic Mean of the $n$ quantities $a, b, c \ldots$ we can in a similar manner find a series of intermediate means ascending from ${ }_{n} \mathrm{H}_{1}$ up to the Geometric Mean $(a b c \ldots)^{\frac{1}{n}}$.

Let ${ }_{n} Q_{p} \equiv$ the sum of all $p$-combinations of $\frac{1}{a}, \frac{1}{b} \ldots$ and ${ }_{n} \mathrm{H}_{p} \equiv{ }_{n} \mathrm{C}_{p} \div{ }_{n} \mathrm{Q}_{p}$, then ${ }_{n} \mathrm{H}_{2} \frac{1}{{ }^{2}},{ }_{n} \mathrm{H}_{3} \frac{1}{3} \ldots{ }_{n} \mathrm{H}_{n-1}^{\frac{1}{n-1}}$ clearly form such a series.

For the reciprocals of these are related to $a^{-1}, b^{-1}, c^{-1} \ldots$ exactly as ${ }_{n} \mathbf{A}_{2}^{\frac{1}{2}},{ }_{n} \mathbf{A}_{3}{ }^{3} \ldots$ are related to $a, b, c \ldots ;$ so that ${ }_{n} \mathrm{H}_{1}^{-1},{ }_{n} \mathrm{H}_{2}^{-\frac{1}{2}},{ }_{n} \mathrm{H}_{3}^{-\frac{1}{5}} \ldots{ }_{n} \mathrm{H}_{n}^{-\frac{1}{n}}$ give a series of means of $a^{-1}, b^{-1} \ldots$ in descending order, beginning with their arithmetic, and ending with their geometric mean; and therefore ${ }_{n} \mathrm{H}_{1},{ }_{n} \mathrm{H}_{2} \frac{1}{2},{ }_{n} \mathrm{H}_{3}{ }^{\frac{1}{3}} \ldots{ }_{n} \mathrm{H}_{n}^{\frac{1}{n}}$ from an ascending series, from ${ }_{n} \mathrm{H}_{1}$ the harmonic mean of $a, b, c \ldots$ up to ${ }_{n} \mathbf{H}_{n}{ }^{\frac{1}{n}}$ their geometric mean. 
6. If we assume $a \neq b=c=d$... then a slight modification of the investigation in paragraph 3 gives us the result

$$
\begin{aligned}
n^{p+1}\left({ }_{n} \mathbf{A}_{p}{ }^{p+1}-{ }_{n} \mathbf{A}_{p+1}^{p}\right) & =(a-b)^{2} b^{p^{2}-1}\left[u^{p-1}+2 u^{p-2} w+3 u^{p-2} w^{2}+\ldots+p w^{p-1}\right] \\
& \text { when } u \equiv(a-b) p+n b \text { and } w \equiv(a-b p+1)+n b .
\end{aligned}
$$

This suggests that, removing the restriction $b=c=d \ldots$ the value of ${ }_{n} A_{p}^{p+1}-{ }_{n} A_{p+1}^{p}$ may be expressible in the form

$$
\Sigma\left\{(a-b)^{2} \phi(a, b, c \ldots)\right\}
$$

where the summation $\Sigma$ extends over all pairs of the quantities. The following particular cases may be of interest. They have been obtained by a direct and rather laborious application of undetermined multipliers.

$$
\begin{aligned}
& { }_{3} \mathrm{~A}_{2}{ }^{3}-{ }_{3} \mathrm{~A}_{3}{ }^{2}=\frac{1}{54}=\left[(a-b)^{2}\left\{7 a b c^{2}+(a+b) c^{3}\right\}\right]
\end{aligned}
$$

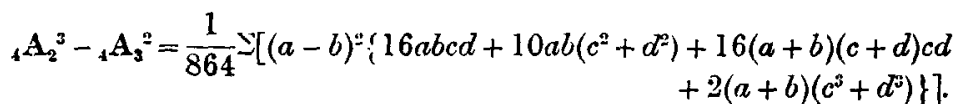

7. More generally

$$
\begin{aligned}
& { }_{n} A_{2}{ }^{3}-{ }_{n} A_{3}{ }^{2}=\frac{2}{n^{3}(n-1)^{3}(n-2)^{2}} \cdot:\left[( a - b ) ^ { 2 } \left\{2(n-2)(a+b) \Sigma c^{3}\right.\right. \\
& +(13 n-20)(a+b) \Sigma c^{2} d+12(5 n-7)(a+b) \Sigma c d e-\left(n^{2}-13 n+16\right) a b \Sigma c^{2} \\
& -4(3 n-4)(u-5) a b \Sigma c d+12(3 n-4) \Sigma c d e f\}]
\end{aligned}
$$

where the summations $\Sigma$ within the square brackets refer to the $n-2$ quantities $b, c \ldots$, (omitting $a$ and $b$ ).

I have not tried to get an expression in a form of this type of the general difference ${ }_{n} \mathrm{~A}_{p^{p+1}}-{ }_{n} \mathrm{~A}_{\mu+1}^{\mu}$, as the investigation would be very laborious by the method applied to find the special cases. It may be noted, however, that the coefficient of $a b \mathrm{I} c d$ in the last formula becomes negative when $n>5$, and that of $a b \vdots c^{\prime \prime}$ when $n=11$, and a consideration of the special case $a \neq b \neq c=d=e \ldots$ has convinced me that the coefficient of $(a-b)^{2}$ as a whole, is not necessarily positive, though our previous general investigation showed that ${ }_{n} A_{2}{ }^{3}-{ }_{n} A_{3}{ }^{2}$ must be positive.

If the value of ${ }_{n} A_{p}^{p+1}-{ }_{n} A_{p+1}^{p}$ could be thrown into a form which would be manifestly positive, we should thereby obtain a direct proof of the main theorem of this section, without the use of Mathematical Induction. 
8. For two quantities it is well known that the product of the Arithmetic and Harmonic Means is equal to the square of the Geometric Mean; but this does not hold for $n$ quantities. Does it hold for any pair of corresponding means ${ }_{n} \mathrm{~A}_{\mu^{j}}^{\frac{1}{\mu}}$ and ${ }_{n} \mathrm{H}_{\mu}^{\frac{\mathrm{T}}{\nu}}$ ?

It is easily seen that the product of these is greater or less than the square of the Geometric Mean according as (leaving out prefixes to $\mathrm{A}, \mathrm{H}, \mathrm{P}$ )

$$
\begin{aligned}
& \mathrm{A}_{p} \mathrm{H}_{p} \text { is }>\text { or }<\mathrm{P}^{\frac{2 p}{n}} \\
& \text { or as } \mathrm{P}_{p} \mathrm{P}_{n}>\text { or }<\mathrm{P}^{\frac{2 p}{n}} \mathrm{P}_{n-p} \\
& \text { or as } \mathrm{P}_{p}{ }^{n} \mathrm{P}_{n}{ }^{n-2 p}-\mathrm{P}_{n-p}^{n} \text { is }>\text { or }<0 .
\end{aligned}
$$

The last expression becomes $=0$ when $p=n / 2$, which of course is possible only when $n$ is even. To test whether the expression has a definite sign for given values of $n, p$, independent of the values of $a, b \ldots$, I have tried the particular case $a \neq b=c=d=e=\ldots$, in which case it has the same sign as

$$
\left(\frac{a}{b}\right)^{n-2 p}\left(p \frac{a}{b}+n-p\right)^{n}-\left\{(n-p) \frac{a}{b}+p\right\}^{n}
$$

This shows that excepting when $p=n / 2$, the sign is not definite but depends on the values of $a, b, \ldots$.

Thus when $n$ is even, one pair of the means $H_{p}^{\frac{1}{p}}, A_{p}^{\frac{1}{p}}$ has the same geometric mean as $a, b, c \ldots$, namely, that for which $p=n / 2$.

9. Two other sets of means, in continually descending order from the Arithmetic to the Geometric, and from the Geometric to the Harmonic Mean respectively, are given by the quantities

$$
\text { and } \begin{array}{r}
\mathrm{G}^{2} \div \mathrm{H}_{\mu^{\prime \prime}}^{\prime \prime},(p=1,2,3 \ldots u) \\
\mathrm{G}^{2} \div \mathbf{A}_{\mu \prime \prime}^{\prime \prime},(p=1,2,3 \ldots u)
\end{array}
$$

where $\mathrm{G}$ is the gcometric mean of $a, b, \ldots$; and many other means might be invented by compounding these in other ways.

II.

10. All the means in Section I. lie between the Arithmetic and the Harmonic Means; and for a given value of $n$ their number is limited. Those in the present section are unlimited in number, and they extend riglit up to the greatest and down to the least of the 
quantities $a, b, c \ldots$ As the types are independent of $n$ we shall first take the simplest case of two quantities $a$ and $b$, and define the mean $\mathrm{M}(p)$ by

$$
\begin{aligned}
\mathrm{M}(p) & \equiv\left(\frac{a^{p}+b^{\mu}}{2}\right)^{\frac{1}{p}} \\
\text { so that } \quad \mathrm{M}\left(\frac{1}{p}\right) & \equiv\left(\frac{a^{\frac{1}{p}}+b^{\frac{1}{p}}}{2}\right)^{p}
\end{aligned}
$$

and in the first instance we shall keep $p$ integral.

11. It is well known that $M(1), M(2), M(3) \ldots$ are in ascending order, and $M(1), M\left(\frac{1}{2}\right), M\left(\frac{1}{3}\right) \ldots$ in descending order, and also that $\mathbf{M}(-1), \mathbf{M}(-2), \mathbf{M}(-3) \ldots$ are in descending and $\mathbf{M}(-1), \mathbf{M}\left(-\frac{1}{2}\right)$, $\mathbf{M}\left(-\frac{1}{3}\right) \ldots$ in ascending order. It is obvious that $\mathbf{M}(1)$ is the Arithmetic, and $\mathrm{M}(-1)$ the Harmonic Mean.

12. Again $\mathrm{M}(p) \times \mathrm{M}(-p)=\left(\frac{a^{p}+b^{p}}{2}\right)^{\frac{1}{p}} \div\left(\frac{a^{-p}+b^{-p}}{2}\right)^{\frac{1}{p}}$

$$
=\left(\frac{a^{p}+b^{p}}{2} \times \frac{2 a^{p} b^{\mu}}{b^{p}+a^{p}}\right)^{\frac{\mathrm{J}}{p}}=a b .
$$

Thus $\mathbf{M}(p)$ and $\mathbf{M}(-p)$ have the same geometric mean as $a, b$.

13. Clearly the limit of $M(p)$ for $p=\infty$ is the same as that of $a\left\{\frac{1+\left(\frac{b}{a}\right)^{p}}{2}\right\}^{\frac{1}{p}}$, which when $a>b$ is easily seen to be $a$.

Similarly the limit of $\mathbf{M}(-p)$ for $p=\infty$ is $b$, the smaller of the two quantities.

14. To find the limit of $\mathrm{M}\left(\frac{1}{p}\right)$ when $p=\infty$ put $c=\sqrt{a \overline{a b}}$ and $y=\sqrt{ } \frac{a}{b}$, so that

$$
\begin{gathered}
\mathrm{M}\left(\frac{1}{p}\right)=c\left(\frac{y^{\frac{1}{p}}+y^{-\frac{1}{p}}}{2}\right)^{p}=c\left(1+\frac{d^{2}}{2}\right)^{p} \\
\text { where } d \equiv y^{\frac{1}{2 p}}-y^{-\frac{1}{2 p}}
\end{gathered}
$$

a quantity which tends towards 0 when $p$ gets large. 
Expanding $\left(1+\frac{d^{2}}{2}\right)^{p}$ by the Binomial Theorem, it at once appears that the limit is 1 , if that of $p d^{2}$ is zero.

Now $\quad p d^{2}=p\left(\frac{1}{y^{2 p}}-y^{\frac{-1}{2 p}}\right)^{2}=p\left\{\left(\frac{\log y}{2 p}\right)+\frac{1}{\mid \underline{3}}\left(\frac{\log y}{2 p}\right)^{3}+\ldots \ldots\right\}^{2}$ and this has clearly zero as limit, for $p=\infty$.

Thus the limit of $M\left(\frac{1}{p}\right)$ for $p=\infty$ is $\sqrt{a \bar{b}}$. And since $M\left(\frac{1}{p}\right) \cdot M\left(-\frac{1}{p}\right)=a b$ it follows that the limit of $\mathrm{M}\left(-\frac{1}{p}\right)$ for $p=\infty$ is also $\sqrt{a \bar{b}}$

15. The results of paragraphs $12-15$ may be tabulated thus :--

\begin{tabular}{|c|c|c|c|c|c|}
\hline$p$ & $\infty$ & 1 & 0 & -1 & -8 \\
\hline $\mathbf{M}(p)$ & $a$ & $\frac{a+b}{2}$ & $\sqrt{a b}$ & $\frac{2 a b}{a+b}$ & $b$ \\
\hline
\end{tabular}

We shall find that excepting the result of 12 , these properties can be extended to $n$ quantities $a b c . .$.

16. As in Chrystal's Algebra, Chapter xxiv., Art. 9, we can prove $\quad\left(\frac{a^{m}+b^{m}+c^{m}+\ldots}{n}\right)^{\frac{I}{m}}>\frac{a+b+c+\ldots}{n}$ when $m>1$.

Hence putting $m=\frac{p+1}{p}$ and writing $x$ for $a^{\frac{1}{p}}$ and transforming, we get $\left(\Sigma x^{p+1} / n\right)^{\frac{1}{p+1}}>\left(\Sigma x^{\nu} / n\right)^{\frac{1}{p}}$ which shows that $M(1), M(2), M(3) \ldots$ are in ascending order, where $\mathbf{M}_{p} \equiv\left(\frac{a^{p}+b^{p}+c^{p}+\ldots}{n}\right)^{\frac{1}{p}}$

Again, writing $y$ for $a^{p+1}$, we have

$$
\left(\Sigma y^{\frac{1}{p}} / n\right)^{p}>\left(\Sigma y^{\frac{1}{p+1}} / n\right)^{p+1}
$$

which shows that $M(1), M\left(\frac{1}{2}\right), M\left(\frac{1}{3}\right) \ldots$ are in descending order. 
17. Since $M(-p)$ is the reciprocal of $\left(\frac{\Sigma a^{-p}}{n}\right)^{\frac{1}{p}}$, which bears the same relation to $a^{-1}, b^{-1}, \ldots$ as $\mathbf{M}(p)$ does to $a, b \ldots$ it follows that $\mathbf{M}(-p)$ diminishes as $p$ increases from 0 to $\infty$.

18. As in the case of two quantities, we easily prove that Limit $\mathbf{M}(p)=a$ the greatest of the quantities, while Limit $\mathbf{M}(-p)=b$, the least of the quantities $a, b, \ldots$.

It remains to prove that $\operatorname{Limit}_{p=0} \mathbf{M}(p)=(a b c \ldots)^{\frac{1}{n}}$.

19. If $n=2^{k}$, we have

$$
\begin{aligned}
a+b+c+\ldots= & \left(a^{\frac{1}{2}}-b^{\frac{1}{2}}\right)^{2}+\left(c^{\frac{1}{2}}-d^{\frac{1}{3}}\right)^{2}+\ldots \\
& +2\left(a^{\frac{1}{4}} b^{\frac{1}{4}}-c^{\frac{1}{4}} d^{\frac{1}{4}}\right)^{2}+\ldots+\ldots \\
& +4\left(a^{\frac{1}{x}} b^{\frac{1}{8}} c^{\frac{1}{5}} d^{\frac{1}{x}}-e^{\frac{1}{x}} f^{\frac{1}{*}} g^{\frac{1}{s}} h^{\frac{1}{5}}\right)^{2}+\ldots \\
& +\ldots \ldots \\
& +\frac{n}{2}\left(a^{\frac{1}{4}} b^{\frac{1}{n}} \ldots-u^{\frac{1}{n}} v^{\frac{1}{4}} \ldots\right)^{2} \\
& +n(a b c \ldots)^{\frac{1}{n}} \\
= & \text { squared differences }+n(a b c \ldots)^{\frac{1}{n}}
\end{aligned}
$$

If $n \neq 2^{k}$ let $n+v=2^{k}$, then

$$
\begin{aligned}
a+b+c+\ldots+ & (g+g+g \ldots \text { to } v \text { terms }) \\
& =\text { squared differences }+(n+v)(a b c \ldots g g g \ldots)^{-\frac{1}{n+v}}
\end{aligned}
$$

Putting $g=(a b c \ldots)^{\frac{1}{n}}$ we get

$$
\begin{aligned}
& a+b+\ldots+v g=\text { squared differences }+(u+v)\left(g^{n} g^{\nu}\right)^{\frac{1}{n+\nu}} \\
& \therefore a+b+c \ldots-\text { squared differences }+n g .
\end{aligned}
$$

Hence we have

$$
\begin{aligned}
& a^{\frac{1}{p}}+b^{\frac{1}{p}}+\ldots=\left(a^{-\frac{1}{2 p}}-\frac{1}{b^{2 p}}\right)^{2}+\ldots+\ldots \\
& +2\left(a^{\frac{1}{4 p}} \frac{1}{b^{4 p}}-c^{\frac{1}{4 p}} d^{\frac{1}{4 p}}\right)^{2}+\ldots+\ldots \\
& +\ldots \ldots \ldots+n \frac{1}{n g^{\prime \prime}}
\end{aligned}
$$


Thus

$$
\begin{aligned}
& \left(\frac{a^{\frac{1}{\mu}}+b^{\frac{2}{\mu}}+\ldots}{n}\right)^{p}=\left(g^{\prime}+\delta\right)^{\prime \prime} \\
& \text { when } \hat{\delta}=\frac{1}{n}\left\{\left(a^{\frac{1}{2^{\prime \prime}}}-b^{\frac{1}{2 \mu}}\right)^{2}+\ldots\right\}
\end{aligned}
$$

a quantity which clearly gives $\operatorname{Limit}_{p=\infty} p \delta=0$.

Hence as in the case of only two quantities, we find

Limit of $\mathrm{M}\left(\frac{1}{p}\right)$ for $p=\infty$ is the Geometric Mean of $a b c \ldots$.

Thus the means indicated by $\mathbf{M}(p)$ completely fill up the interval from the lowest to the highest of the quantities $a b c \ldots$ as $p$ increases from $-\infty$ up to $\infty$, and they have the same Geometric Mean as their value for $p=0$, the Arithmetic Mean for $p=1$, and the Harmonic Mean for $p=-1$.

20. As in 9 , we could define a cognate set of means $\mathrm{N}(p)$ by the relation $\mathrm{M}(p) . \mathrm{N}(p)=\mathrm{G}^{2}$ where $\mathrm{G}$ is the geometric mean.

21. In Chrystal's Algebra, Chap. xxIv., Exercises v., there is a reference to Schlömilch-Zeitschrift für Mathematik, Vol. III., p. 301, from which it would appear that some of the properties of the means M. were long ago worked out by Schlomilch; but if the notation in "Chrystal" is accurate, the full symmetry of the subject does not seem to have been exhausted by Schlömilch.

The subject of Algebraic Means is obviously one capable of indefinite development, but probably this paper is long enough. I may mention that $I$ have found some general inequality theorems relating to means (amongst others) involving powers of sums of the letters taken $r$ at a time. 